\title{
Embedded high resolution sensor based on optical feedback in Vertical Cavity Surface Emitting Laser
}

\author{
Bram Van Hoe, Deben Lamon \\ Supervisor(s): Peter Van Daele, Geert Van Steenberge
}

\begin{abstract}
This paper describes the fabrication of a novel type of pressure sensor based on optical feedback in a Vertical Cavity Surface Emitting Laser (VCSEL). The detection mechanism of the sensor is based on a displacement measurement through self-mixing interferometry in the laser cavity. The use of unpackaged VCSELs enables the embedding in thin flexible optical foils which results in an integrated pressure sensor. A proof of principle set-up has been developed and a first integrated sensor is tested.
\end{abstract}

Keywords-Integration, Optical feedback, Optical sensing, Self-mixing interferometry, VCSEL

\section{INTRODUCTION}

Sensors to measure physical properties such as pressure, temperature and the proximity to other objects are widespread and developed by multiple companies and research centers. They are mostly based on piezoresistive, piezoelectric or capacitive force sensing technologies.

The sensor we present however is an optical alternative based on the use of VCSELs as pressure sensitive elements. Using unpackaged laser diodes, one can integrate this sensor in a thin optical package (an optical sensing foil) which is flexible and can be mounted on a non-planar surface or even flexible objects, such as a human body.

The three unique advantages of this sensor are the sensitivity (typically down to $425 \mathrm{~nm}$, in case of a VCSEL wavelength of $850 \mathrm{~nm}$ ), the resolution (depending on the pitch of the VCSEL array, typically a few hundred $\mu \mathrm{m}$ ) and the embedding possibilities in optical foils.

B. Van Hoe is with the Center for Microsystems Technology (CMST), Ghent University (Ugent), Gent, Belgium. E-mail: Bram.VanHoe@UGent.be.

\section{SENSING MECHANISM}

The pressure sensing mechanism is based on the self-mixing interference effect in VCSELs which is observed when a fraction of light emitted from the laser is injected back into the laser cavity by reflection from an external target [1], as depicted in figure 1 . The reflected light will be superimposed with the light inside the laser cavity depending on the phase shift introduced by the round trip travel to and from the target.

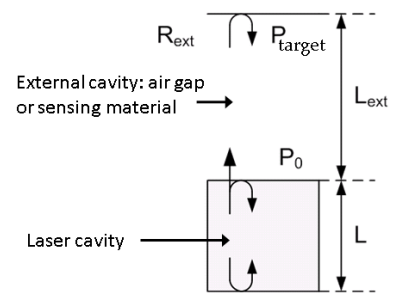

Figure 1. VCSEL sensing principle: applying a pressure will change the external cavity length, $L_{\text {ext }}$

This will result in a periodic variation of wavelength, optical power, and electrical steering voltage/current, all with period $\lambda / 2$ ( $\lambda$ being the wavelength of the VCSEL, $850 \mathrm{~nm}$ ). This means that the target displacement can be calculated by counting the number of peaks between the initial and the final position of the target.

\section{PROOF OF PRINCIPLE}

A discrete set-up is developed to provide a proof of principle of this new sensing mechanism: a VCSEL is positioned on a fixed location and a moveable reflector is positioned sufficiently close to the active area. The cavity between the VCSEL and the reflector consists of 
an air gap and the position of the external reflector, consisting of a Au coated fiber facet, is changed by micro stages. The length of the air gap is consequently varied by changing the position of the external reflector.

Variations in both electrical current and optical power are monitored simultaneously. Both steering of the reflector and read-out of the sensor are driven by a computer. This set-up has proven the sensing mechanism both in singleand multimode operation of the VCSEL. Periodic variations were observed in the optical power as well as in the electrical current. The comparison between the periodicity in electrical current without (REF) and with (EXP) optical feedback is shown in figure 2 .

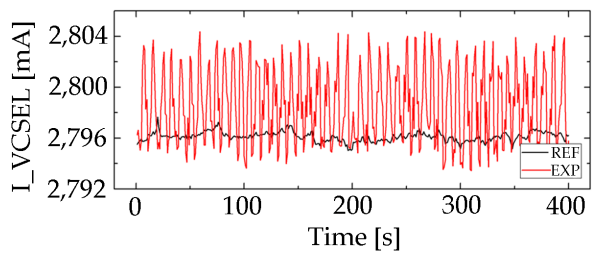

Figure 2. Discrete set-up: electrical current $\left(\mathrm{V}_{\mathrm{VCSEL}}=1,6 \mathrm{~V}\right)$

\section{INTEGRATION OF THE SENSOR}

When embedding this sensor in an optical foil, the external air gap cavity has to be replaced by a thin, compressible sensing layer.

The processing of the integrated pressure sensor [2] starts on a rigid FR-4 substrate with a copper layer that serves as a heat sink for the VCSEL. This substrate is covered by an SU-8 layer and a cavity is made by laser ablation to position the VCSEL. A final $10 \mu \mathrm{m}$ thick SU-8 layer is spincoated on top and micro-vias to the VCSEL contacts are created by laser ablation. Finally, the sensor layer is applied by spinning Sylgard ${ }^{\circledR} 184$, a thermally curable PDMS material [3], with a thickness of $100 \mu \mathrm{m}$ (figure 3).

First characterization tests of this sensor, monitoring both optical power and electrical current, have been carried out and confirm the proposed sensing mechanism. An example of the periodic electrical current variations as a result of the applied force is shown in figure 4.

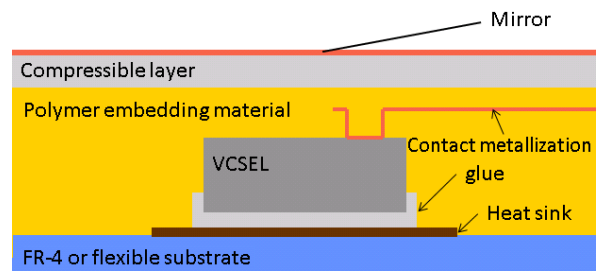

Figure 3. Schematic view of the integrated sensor

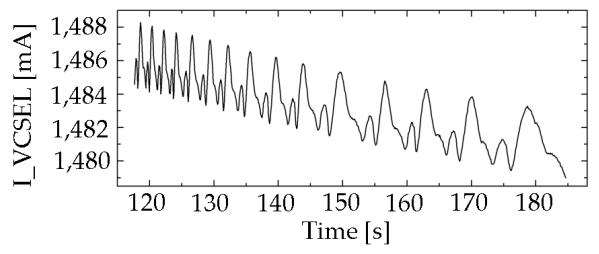

Figure 4. Integrated VCSEL: electrical current $\left(\mathrm{V}_{\mathrm{VCSEL}}=1,8 \mathrm{~V}\right)$

\section{CONCLUSIONS}

The integrated optical sensor, based on optical feedback in a VCSEL, can be used in several applications where a high density and high accuracy sensor is needed. A proof of principle set-up has proven the sensing mechanism and the embedding techniques have shown the integrating possiblities.

\section{ACKNOWLEDGMENTS}

This work is partially conducted in the framework of the project Flexible Artificial Optical Skin (FAOS), and is supported in part by the Institute for the Promotion of Innovation through Science and Technology (IWT), Flanders, Belgium. B. Van Hoe is also supported by a $\mathrm{PhD}$ grant of the IWT.

\section{REFERENCES}

[1] Y. L. Lim, K. Bertling, P. Rio, J. R. Tucker, A. D. Rakic, "Displacement and distance measurement using the change in junction voltage across a laser diode due to the self-mixing effect", Proceedings of SPIE, Photonics: Design, Technology, and Packaging II, vol. 6038, pp. 378-387, Jan 2006.

[2] E. Bosman, G. Van Steenberge, N. Hendrickx, J. Vanfleteren, P. Van Daele "Active optical links embedded in flexible substrates", Proceedings of 58th Electronic components and technology conference, pp. 1150-1157, May 2008.

[3] Dow Corning, http://www.dowcorning.com 